\title{
Fibrosis quística: generalidades
}

\section{Cystic fibrosis: generalities}

Pág. 20,33

Recibido: 16-06-2020

Aceptado: 26-08-2020

Dr. Eliécer Antonio Cordero Araya ${ }^{1}$

Dra. Yelín Choque Núñez ${ }^{2}$

Dra. María José Quirós Chacón ${ }^{3}$

1,2. Trabajador independiente, Cartago Costa Rica

\section{RESUMEN}

La fibrosis quística, es una enfermedad genética autosómica recesiva, que es causada por mutaciones en un gen ubicado en el cromosoma 7 y que codifica una proteína denominada Regulador de Conductancia Transmembrana de la Fibrosis Quística, ella está ubicada en numerosos epitelios, de ahí la afectación multisistémica, sobre todo en sistema respiratorio, gastrointestinal y genitourinario.

El diagnóstico por lo general se realiza o se sospecha mediante el cribado neonatal y se confirma por estudios como la medición de cloruro en sudor. El propósito en el tratamiento se basa en disminuir los síntomas, evitar complicaciones y mejorar la calidad de vida. Los avances tanto en el diagnóstico y el tratamiento sobre todo de la infección pulmonar y nutricional, han provocado gran mejoría en la sobrevida de estos pacientes, por lo tanto, actualmente no se considera solo una enfermedad de edad pediátrica, sino también una enfermedad crónica que afecta a adultos.

\section{PALABRAS CLAVE:}

fibrosis quística, mutaciones, secreciones, multisistémica, tratamiento.

\section{ABSTRACT}

Cystic fibrosis, is an autosomal recessive genetic disease, which is caused by mutations in a gene located in the chromosome 7 and that encodes a protein called Transmembrane Conductance Regulator of cystic fibrosis, it is located in numerous epithelia, hence the multisystem involvement, especially in the respiratory, gastrointestinal and genitourinary systems. The diagnosis is usually made or suspected through neonatal screening and is confirmed by studies such as the measurement of chlorine in sweat. The purpose of treatment is based on reducing symptoms, avoiding complications 
and improving quality of life. Advances in both the diagnosis and treatment, especially of pulmonary and nutritional infections, have led to great improvement in the survival of these patients; therefore, it is not currently considered only a disease of pediatric age, but also a chronic disease that affects to adults.

\section{KEYWORDS}

Cystic fibrosis, mutations, secretions, multisystemic, treatment.

\section{INTRODUCCIÓN}

La fibrosis quística (FQ) es una enfermedad multisistémica que afecta tanto niños como adultos; actualmente es el trastorno genético recesivo que afecta con mayor frecuencia a la población caucásica y su incidencia es de 1 de cada 1800 a 25000 recién nacidos, esto dependiendo de la región o etnia de origen $(1,2)$. Este trastorno fue descrito como nueva entidad clínica desde el año 1936, posterior hacia 1946, se sospechó de su origen genético y finalmente en 1989, se publicó la identificación del gen, el cual se denominó Regulador de Conductancia Transmembrana de la Fibrosis Quística (CFTR) (3). Las características de la población con fibrosis quística han cambiado dramáticamente con el paso de los años, previamente era reconocida como una enfermedad pediátrica con pobre pronóstico, por lo general menor a 5 años; sin embargo, tras los estudios y el progreso realizado en el diagnóstico, manejo y tratamiento de esta, la supervivencia ha alcanzado un aproximado de 40 años a nivel mundial y de hasta 53 años en las personas que habitan países desarrollados $(3,4,5)$. El presente artículo tiene por objetivo realizar una revisión bibliográfica general acerca del tema de fibrosis quística, incluyendo bases genéticas, manifestaciones clínicas, diagnóstico y tratamiento; con el fin de facilitar su actualización en el gremio médico.

Método

El artículo de revisión se elaboró mediante amplia consulta de múltiples fuentes bibliográficas, de las cuales se seleccionaron 20 por reunir los requisitos de contener información precisa y relevante del tema de fibrosis quística y estar publicadas en el periodo de años 2015-2020; se hallaron 14 referencias en el idioma inglés y 6 en español. Las bases de datos utilizadas fueron Up to date, Dynamed y PubMed; además, se consultó las últimas ediciones de los libros Principios de Medicina Interna de Harrison y Libro de Texto de Pediatría de Nelson. Los términos usados para la búsqueda de información fueron: fibrosis quística, enfermedades genéticas en pediatría y test del sudor.

\section{GENÉTICA}

La FQ es una enfermedad autosómica recesiva, es decir; requiere mutaciones patógenas en ambas copias del gen para expresar enfermedad clínica, y se produce tras la mutación de un gen ubicado en el brazo largo del cromosoma 7, que codifica la producción de una proteína denominada Regulador de Conductancia Transmembrana de Fibrosis Quística (CFTR) por sus siglas en inglés (Cystic Fibrosis Transmembrane Regulator); esta proteína funciona como un canal para el paso del ión cloruro principalmente y se encuentra presente en múltiples epitelios $(6,7,8)$.

Se han descubierto más de 2000 mutaciones del gen CFTR capaces de provocar patología y se diferencia entre ellas por cambios puntuales mínimos $(4,7)$. La primera mutación descrita, 
a su vez la más frecuente, es F508del (la nomenclatura indica la omisión de un solo residuo fenilalanínico [F] en posición 508 del CFTR) y esta se observa aproximadamente en un $50 \%$ de los cromosomas estudiados; sin embargo, su frecuencia varía entre los distintos grupos étnicos, al igual que el resto de mutaciones (4, $7,8)$.

Las mutaciones del gen CFTR pueden clasificarse en 6 grupos funcionales según su efecto a nivel de la proteína, clase I: ausencia de síntesis de CFTR, clase II: maduración defectuosa y degradación prematura de las proteínas (a este grupo pertenece la Phe508del); clase III: defecto en la apertura o regulación del canal, clase IV: conductancia defectuosa por el poro del conducto iónico, clase $\mathrm{V}$ : disminución del número de transcriptos de CFTR por una anomalía de un promotor o empalme (síntesis de proteína reducida), clase VI: recambio acelerado desde la superficie celular (vida media reducida) $(4,7,8)$. Las mutaciones de clase I, II y III se asocian con formas clínicas más severas como enfermedad pulmonar grave e insuficiencia pancreática y el resto de grupos están ligados a formas clínicas más leves, ya que los tipos IV, $\mathrm{V}$ y $\mathrm{VI}$ corresponden a un defecto parcial de la proteína $\operatorname{CFTR}(4,7,9)$.

\section{PATOGÉNESIS}

EICFTResunaproteínaintegraldemembranaque actúa como conducto, facilita específicamente el transporte de cloruro, a su vez bicarbonato, sodio y agua; lo cual permite la movilización y eliminación de productos exocrinos (8). Los cambios generados por el mal funcionamiento del CFTR, dan lugar a la escasez de agua en las secreciones mucosas, por consiguiente, que sean más espesas; además, provocan falta de limpieza de las secreciones mucosas, contenido elevado nivel de sal en el sudor junto a otras secreciones serosas y finalmente, infección crónica limitada al tracto respiratorio $(1,8)$.

El insuficiente o nulo funcionamiento de esta proteína provoca, por lo tanto, alteraciones en el transporte de los iones a nivel de todas las células donde se expresa; de tal manera que existen manifestaciones clínicas relevantes en el aparato respiratorio, sistema gastrointestinal, genitourinario y glándulas sudoríparas $(4,8)$.

En el caso de la vía aérea, la alteración del transporte iónico produce una deshidratación del líquido periciliar que transforma el moco en una secreción espesa y deshidratada, lo cual evita un correcto aclaramiento mucociliar (1, 4). Las secreciones mucosas, al ser retenidas obstruyen las vías respiratorias, comenzando por las de menor calibre, es decir los bronquiolos, esta corresponde a la primera anormalidad fisiológica observable en el sistema respiratorio (1). Tanto la retención de moco como la falta de aclaramiento, favorecen la colonización bacteriana crónica progresiva, asociada a una respuesta inflamatoria neutrofílica alterada y mal regulada; ambos procesos, la infección y la inflamación crónica, producen un círculo vicioso de destrucción tisular $(1,4,7)$.

Es posible que ocurran eventos fisiopatológicos similares de secreciones espesas, obstrucción y defectos en el aclaramiento (o mala digestión), que conllevan a la enfermedad del tracto gastrointestinal (incluyendo páncreas e hígado) y conductos deferentes $(1,8)$. Debido a que la función del conducto de las glándulas sudoríparas es absorber el cloruro en lugar de secretarlo y el sodio viaja con él, este sodio no se recupera del sudor primario a medida que se transporta a la superficie de la piel; por lo que los niveles de cloruro y sodio son consecuentemente elevados en sudor (1).

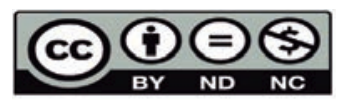




\section{MANIFESTACIONES CLÍNICAS}

Dado a la existencia de gran variedad de mutaciones genéticas que producen la $F Q$, en la literatura se describe manifestaciones multisistémicas causadas por cambios en el gen CFTR; sin embargo, se ha observado que afecta principalmente el sistema gastrointestinal $y$ el respiratorio $(3,10)$.

\section{a. Tracto respiratorio:}

La enfermedad pulmonarsigue siendo la principal causa de morbimortalidad en pacientes con FQ y las manifestaciones son amplias, incluyen desde lactantes asintomáticos hasta pacientes con síntomas y signos como tos persistente, alteración de las pruebas de función pulmonar que traducen enfermedad obstructiva en fases tempranas y un patrón mixto obstructivo y restrictivo en fases avanzadas de la enfermedad $(4,10)$.

El aumento de viscosidad del moco produce la colonización de distintos agentes bacterianos tales como: Staphylococcus aureus, Haemophilus influenzae, Pseudomonas aeruginosa, Klebsiella sp, Stenotrophonomas maltophilia, Burkholderia cepacia, Aspergillus $\mathrm{sp}$, entre otros; en la mayoría de los pacientes esta colonización es polimicrobiana, incluyendo otros microorganismos como hongos o bacterias atípicas. En la infancia, el patógeno que produce con mayor frecuencia la colonización patógena es el Staphylococcus aureus, posteriormente conforme el paciente se hace mayor aumenta la colonización por Pseudomonas aeruginosa, el cual es el microorganismo más frecuentemente aislado en la edad adulta (9). Como consecuencia de las condiciones en el epitelio pulmonar, la Pseudomonas aeruginosa puede generar cambios fenotípicos comunes como la hipoxia local y puede producir grandes colonias bacterianas conocidas como biofilms, donde las bacterias se mantienen inactivas, siendo complicada su eliminación $(7,9)$.

Las exacerbaciones se presentan por cambios en el patrón usual respiratorio, caracterizado por aumento de tos, dificultad respiratoria, disnea, taquipnea, malestar general, anorexia, aumento de la producción de esputo y cambios en las características de las secreciones; por otro lado las infecciones recurrentes causan lesión a las paredes bronquiales con la consecuente evolución a bronquiectasias y fibrosis, por lo tanto, con cada exacerbación respiratoria se disminuye la función pulmonar $(9,10)$. Otras complicaciones respiratorias que pueden presentar los pacientes con fibrosis quística incluyen: hemoptisis, neumotórax espontáneo, aspergilosis broncopulmonar alérgica e hipertensión pulmonar (11); además, a nivel de vía aérea superior se pueden observar manifestaciones nasosinusales, como aparición de pólipos nasales, obstrucción nasal, mucocele, otitis media y rinosinusitis crónica $(4,9,10)$.

\section{b. Páncreas:}

Se puede alterar de diferentes formas. En los casos más graves, la afectación del páncreas inicia entre las semanas 28 y 32 de gestación por medio del impacto en el desarrollo acinar, conforme pasan los años la destrucción pancreática avanza hasta transformar los acinos en tejido fibroso, cambiando la estructura normal pancreática, lo que provoca deterioro en la producción de enzimas digestivas, manifestado por medio de malabsorción de grasas, proteínas y vitaminas liposolubles $A, D, E, K(8,12)$. La insuficiencia pancreática exocrina está presente desde el nacimiento en dos tercios de los pacientes y otro porcentaje la desarrolla durante la infancia (10). 
Entre las manifestaciones clínicas más comunes seencuentranaquellas causadas porlaabsorción insuficiente de macro y micronutrientes, por lo tanto, retraso del crecimiento, desnutrición y también se expresan deficiencias de vitaminas liposolubles como coagulopatía o raquitismo; la pancreatitis se desarrolla en aproximadamente $10 \%$ de la población con fibrosis quística (10, 13).

Las secreciones espesas disminuyen su flujo produciendo obstrucción de la luz pancreática lo cual interfiere con la adecuada secreción de enzimas, asociado a lisis celular con la consecuente insuficiencia del órgano (14). Así mismo, los pacientes con insuficiencia pancreática exocrina pueden desarrollar disfunción endocrina debido a la destrucción de islotes pancreáticos que conlleva a intolerancia a la glucosa y diabetes relacionada con la fibrosis quística; hasta un $50 \%$ de la población adulta afectada por esta enfermedad, desarrollan este tipo de diabetes $(10,14)$.

\section{c. Tracto gastrointestinal:}

El Íleo meconial, se debe a la pérdida de fluidez del meconio, el cual se ha observado en aproximadamente $10 \%$ de los neonatos (10). La obstrucción intestinal distal puede aparecer en niños y adultos, tiene una prevalencia de hasta $15 \%$ y es más frecuente en pacientes con mutaciones más severas de la enfermedad, esta complicación se observa por obstrucción parcial o total, de la luz intestinal en el íleon terminal o el colon proximal por material intestinal espeso $(10,12)$.

La enfermedad hepatobiliar se puede manifestar por medio de alteraciones en las pruebas de función hepática, hepatomegalia, esteatosis, hasta cirrosis, hipertensión portal y sangrado digestivo por várices (10). Se ha estudiado sobre la pérdida de ácidos biliares por las heces, por lo cual podría existir también colelitiasis a causa de producción de bilis litogénica.

La enfermedad por reflujo gastroesofágico resulta ser más frecuente en pacientes con fibrosis quística, con una prevalencia entre 35$80 \%$ (12). Varios mecanismos contribuyen al reflujo gástrico en pacientes con fibrosis quística, entre los cuales se encuentra: relajación del esfínter esofágico inferior, aumento de la presión intraabdominal por tos crónica, incremento de la acidez gástrica, retraso en el vaciamiento gástrico, entre otros (14).

Otras complicaciones menos frecuentes incluyen el prolapso rectal, estreñimiento, la intususcepción intestinal, patología apendicular, enfermedad inflamatoria intestinal, prevalencia aumentada de colonización crónica por Clostridium difficile y mayor riesgo de cáncer en el tracto gastrointestinal $(12,14)$. El estreñimiento y el prolapso rectal se observa en la actualidad sobre todo cuando existe un tratamiento enzimático inadecuado $(1,10)$.

\section{d. Sistema genitourinario}

Existe fallo en el desarrollo del conducto de Wolffo conducto mesonéfrico durante la embriogénesis, causando defectos en el transporte de esperma por ausencia del conducto deferente, por lo que se observa esterilidad en los hombres, sin embargo, la espermatogénesis y función sexual se encuentran conservadas $(1,10)$. La fertilidad en mujeres se encuentra disminuida, en especial en pacientes con enfermedad pulmonar avanzada, desnutrición y producción de moco cervical más viscoso. Además, se ha 
observado incontinencia urinaria en niños y adolescentes en un 18-47\%, probablemente por causa de sintomatología respiratoria como la tos (1).

\section{e. Sistema óseo}

Múltiples factores contribuyen a la afectación ósea, entre los cuales se encuentra la malabsorción de vitaminas y minerales esenciales, enfermedad hepatobiliar, enfermedad pulmonar avanzada, desnutrición, deficiente actividad física, hipogonadismo y uso crónico de esteroides (13). La densidad ósea se ve disminuida en un $30 \%$ en todos los pacientes con fibrosis quística y hasta un $75 \%$ en pacientes de edad adulta, por esta razón estos pacientes presentan mayor riesgo de deformidades osteomusculares, fracturas óseas y cifosis (10).

\section{Diagnóstico}

Durante los últimos años, el tamizaje neonatal ha detectado a la mayoría de los pacientes diagnosticados con FQ; sin embargo, cada caso amerita la confirmación con el análisis de ADN de la mutación de CFTR y las mediciones de electrolitos en el sudor como pruebas cardinales del diagnóstico $(4,8)$.

Los criterios diagnósticos para la FQ son los siguientes: síntomas y signos típicos de fibrosis quística, exámenes positivos en el cribado neonatal, historia de $F Q$ en hermanos, dos concentraciones de cloruro en sudor mayor $o$ igual a $60 \mathrm{mmol} / \mathrm{L}$ obtenidas en días separados, identificación de dos mutaciones de la proteína CFTR causantes de FQ y divergencia anormal del potencial nasal $(1,10)$.

El cribado neonatal se inicia entre el tercer y séptimo día desde el nacimiento, se utiliza la medición de tripsinógeno inmunoreactivo (IRT), el cual es un precursor de la enzima tripsina y se encuentra generalmente en todos los recién nacidos en pequeñas cantidades; sin embargo, los pacientes con $\mathrm{FQ}$ presentan niveles elevados de este $(2,5)$. El tripsinógeno inmunoreactivo es la prueba inicial en la mayoría de los protocolos, en caso de que esta resulte positiva, se realiza otra prueba de tripsinógeno inmunoreactivo o un análisis de ADN; para esto existen diversos protocolos, entre los cuales se encuentran IRT+IRT, IRT+ADN, IRT+IRT+ADN. $(5,10)$.

Como ya se mencionó antes, si el paciente resulta con IRT elevada, se procede a realizar el análisis de ADN, para lo cual existen los siguientes resultados (2):

- Hallazgo de dos mutaciones, en este caso se realiza un estudio de segregación del ADN para valorar si cada mutación es procedente de uno de los padres del paciente, además se realiza la prueba del sudor.

- Existencia de una sola mutación, se procede a realizar la prueba del sudor. Esta prueba puede resultar incierta, para lo cual se debe ampliar el estudio genético con el fin de buscar una segunda mutación; también podría resultar negativa, si fuese así el paciente sería portador de la enfermedad.

- No se hallan mutaciones y presentan pruebas de sudor normales, se traduce como un falso positivo del examen.

- Pacientes que solo mostraron una mutación en el estudio genético y valores intermedios de la pruebadelsudorolosquepresentanunamutación en cada alelo, pero solo uno es causante de la enfermedad y además, presentan valores de la prueba del sudor normales, se incluyen en un 
grupo por aparte al cual se le conoce como dos términos, sin embargo, resultan ser equivalentes entre ellos: diagnóstico no concluyente de pacientes con examen positivo por fibrosis quística (siglas en inglés: CFSPID) utilizado en varios países y el Síndrome metabólico relacionado con CFTR (siglas en inglés: CRMS) término utilizado en Estados Unidos $(5,10)$. Los pacientes categorizados en este grupo deben ser seguidos de forma regular, puesto que en un futuro podrían desarrollar la enfermedad.

La prueba del sudor corresponde a su recolección, mediante estimulación de la iontoforesis con pilocarpina, posteriormente se realiza una medición de cloruro en la muestra; la clasificación es la siguiente: si el valor es $<30 \mathrm{mmol} / \mathrm{L}$ la prueba es normal, si es de 30$59 \mathrm{mmol} / \mathrm{L}$ es intermedia, en este caso se debe repetir la prueba dos semanas después, si es mayor o igual a $60 \mathrm{mmol} / \mathrm{L}$ la prueba es positiva $(2,5,10,15)$. Se debe realizar antes de las dos semanas de nacido el paciente y cuando él pese más de $2 \mathrm{~kg}(2,5,10,15)$. Esta prueba tiene una sensibilidad del $90-99 \%$, si se realiza de forma adecuada, además posee un alto nivel de especificidad, lo que convierte este examen en el gold standard para el diagnóstico de la FQ $(5,15)$.

Otras pruebas que podrían ser útiles en pacientes con un diagnóstico no definitivo son la diferencia de potencial nasal, análisis de ADN extendido, medición de la función pancreática mediante la elastasa fecal, valores vitamínicos, porcentaje de grasa en heces, pruebas de función pulmonar, entre otros (10).

\section{TRATAMIENTO}

El tratamiento va dirigido a los múltiples órganos y sistemas que se ven afectados por esta enfermedad; además, es indispensable que los pacientes estén a cargo de un equipo multidisciplinario $(6,16)$. Se aborda el tema de tratamiento en dos puntos relevantes: el tratamiento respiratorio y el tratamiento nutricional; los cuales son clave para la disminución de la morbimortalidad en estos pacientes.

\section{a. Tratamiento Respiratorio}

\section{a. a Técnicas que promueven el aclaramiento mucociliar:}

Tiene dos pilares fundamentales, los cuales son: medios físicos para ayudar a la eliminación de secreciones y medicamentos inhalados para disminuir la viscosidad de las secreciones respiratorias (11). Dentro de los medios físicos se encuentra la fisioterapia respiratoria, la cual es una medida que se debe adoptar incluso en pacientes asintomáticos, tiene como objetivo eliminar las secreciones espesas de las vías respiratorias, para mantener la función pulmonar y disminuir el riesgo de infecciones $(4,9,16)$. Adicionalmente, se recomienda realizar actividad física regularmente ya que promueve la eliminación de secreciones de las vías respiratorias, además de los beneficios ya conocidos del ejercicio regular $(4,6,11,16)$.

Los agentes inhalados que facilitan la eliminación de secreciones son DNasa, solución hipertónica y manitol. La DNasa es una enzima que degrada las cadenas de ADN liberado por la degranulación de los neutrófilos, ha demostrado mejoría en la función pulmonar y disminución de las exacerbaciones pulmonares, su uso se recomienda en pacientes mayores de 6 años con enfermedad moderada a grave $(4,6,9,16)$. La solución salina hipertónica actúa como agente osmótico, no se recomienda su uso en menores de 6 años $(2,6,9,11)$. El manitol tiene el mismo 
principio que la solución salina hipertónica, al ser un agente osmótico, es el tratamiento de segunda línea, después de DNasa y solución hipertónica; en el 2019 la FDA aprobó su uso, pero solamente para pacientes adultos con $\mathrm{FQ}$ (11).

\section{a. b Terapia broncodilatadora:}

Además del patrón obstructivo, algunos pacientes con FQ presentan hiperreactividad bronquial $(11,16)$. Por esta razón, se recomienda administrar agonistas beta adrenérgicos de acción corta en las siguientes situaciones (11):

- Inmediatamente antes de las sesiones de fisioterapia respiratoria y ejercicio, para facilitar la depuración de las secreciones.

- Inmediatamente antes de la administración de cualquier medicamento inhalado (solución salina, antibióticos), con el fin de evitar la broncoconstricción generada por estos medicamentos, así como mejorar su distribución y penetración de en la vía aérea.

- Como tratamiento para sualivio en pacientes con hiperreactividad bronquial.

\section{a. c Terapia anti inflamatoria:}

En la $F Q$ existe una inflamación neutrofílica intensa, esto junto a los procesos infecciosos generan destrucción pulmonar (11, 16), por lo que se han descrito medicamentos antiinflamatorios como parte de su tratamiento. Los corticoides inhalados únicamente se recomiendan en pacientes con evidencia de hiperreactividad bronquial, ya que la información de su beneficio es insuficiente $(4,11,16)$, el uso agudo de corticoides sistémicos tiene evidencia limitada en estos pacientes, sin embargo algunos centros recomiendan un curso breve de esteroides, solamente en pacientes que tengan síntomas similares al asma $(16,17)$, el uso crónico de esteroides no está indicado, excepto en pacientes con diagnóstico de asma o aspergilosis broncopulmonar alérgica $(9,11)$. Se sugiere el uso de ibuprofeno a dosis altas, ya que mejora la función pulmonar y disminuye las exacerbaciones, su indicación es en pacientes entre los 6-17 años con una adecuada función pulmonar. $(4,9,11)$.

Con respecto a los macrólidos, su efecto en pacientes con $F Q$ es antiinflamatorio y no antibiótico, su beneficio radica en mejorar la función pulmonar y disminuir las exacerbaciones $(6,9,11)$. Los macrólidos per se no tienen efecto bactericida sobre Pseudomonas aeruginosa; sin embargo, azitromicina ha sido ampliamente estudiado para pacientes con fibrosis quística, ya que tiene un efecto inmunomodulador al reducir la producción de citoquinas proinflamatorias, atenuar los factores de virulencia como la producción de biofilms, además de disminuir la adhesión y migración de neutrófilos $(2,4,16)$.

\section{a. d Tratamiento de las infecciones respiratorias:}

La infección bacteriana crónica de la vía aérea es la principal causa de deterioro de la función pulmonar y aumento de la morbimortalidad en los pacientes con FQ $(6,17)$. Sin embargo, se ha demostrado que el tratamiento oportuno e intensivo de las primeras infecciones y la mejora en los regímenes de tratamiento crónico o de las reagudizaciones, han generado un aumento en la supervivencia y disminución de la morbilidad en pacientes con $F Q$ (4). Con respecto a las vías de administración, la vía oral se prefiere 
para exacerbaciones leves o moderadas; los medicamentos intravenosos se indican cuando hay exacerbaciones graves, fallo o resistencia en el tratamiento oral durante una exacerbación y alergia o intolerancia a los medicamentos orales, mientras que la vía inhalada se usa sobre todo como tratamiento crónico $(4,17)$.

El tratamiento de las infecciones se divide en el tratamiento de las exacerbaciones y el tratamiento crónico.

\section{TRATAMIENTO ANTIBIÓTICO DE LAS EXACERBACIONES:}

El tratamiento de las exacerbaciones se debe basar en su severidad, los resultados del cultivo y la prueba de sensibilidad antibiótica previa o actual, además de tener en cuenta la respuesta al tratamiento en ocasiones anteriores $(6,9)$. Se recomienda el uso de terapia combinada para evitar la aparición de resistencia y beneficiarse del efecto sinérgico de la terapia dual (4). El régimen de tratamiento puede ser ambulatorio o domiciliario, dependiendo de la condición del paciente y de sus comorbilidades, la duración habitual es de 14 a 21 días $(4,9,16)$. En este apartado se hace referencia a las dos bacterias más frecuentes en infecciones asociadas a FQ.

\section{Exacerbaciones leves:}

- Staphylococcus aureus meticilino sensible: trimetoprim sulfametoxazol, amoxicilina más ácido clavulánico $(2,9,17)$.

- Staphylococcus aureus meticilino resistente: trimetoprim sulfametoxazol o linezolid $(2,6)$.

- Pseudomonas aeruginosa: ciprofloxacino o levofloxacino más el antibiótico inhalado de uso habitual; colistina, tobramicina o aztreonam (2, $9,17)$.

\section{Exacerbaciones graves:}

- Staphylococcus aureus meticilino sensible: cefazolina o cloxacilina $(6,17)$.

- Staphylococcus aureus meticilino resistente: linezolid, vancomicina $(2,6,9,17)$.

- Pseudomonas aeruginosa: una beta lactámico (piperacilina-tazobactam, ticarcilinaclavulonato, cefepima, ceftazidima, imipenem, meropenem) más fluoroquinolona (levofloxacino o ciprofloxacino) o aminoglucósido (tobramicina o amikacina) $(6,9,17)$.

Se recomienda tomar una nueva muestra para cultivo de secreción respiratoria, una semana después de concluido el tratamiento, esto para determinar si se logró erradicar la bacteria o si es necesario la continuación de un tratamiento erradicador $(2,17)$.

\section{TRATAMIENTO DE LA INFECCIÓN CRÓNICA:}

La prevalencia de infección crónica por Pseudomonas aeruginosa es elevada en los pacientes con $\mathrm{FQ}$, el objetivo es disminuir lo más posible el número de colonias y no negativizar los cultivos, con esto se evita el deterioro de la función pulmonar y las exacerbaciones $(6,16)$.

Los antibióticos inhalados han facilitado el tratamiento crónico y los más usados son la tobramicina, colistina y aztreonam lisina, se recomienda usar regímenes continuos intercalando los antibióticos sobre todo en pacientes con exacerbaciones frecuentes $y$ deterioro de la función pulmonar y, recordando siempre el uso de beta adrenérgicos de acción 
corta para evitar el broncoespasmo $(6,16,18)$.

\section{PREVENCIÓN DE LAS INFECCIONES}

La inmunización es fundamental en los pacientes con $F Q$, se recomienda la vacunación anual contra la influenza estacional a partir de los 6 meses de edad, también se recomienda la vacunación contra Streptococcus pneumoniae, la 13 valente hasta los dos años y la 23 valente después de los 2 años de edad, aunque la infección por este agente es poco frecuente en estos pacientes, el beneficio es mayor que el riesgo $(11,18)$. Además, se debe de extremar medidas de prevención de infecciones como el distanciamiento en áreas comunes, el uso de cubrebocas en ambientes hospitalarios y una adecuada higiene de manos (18). No se recomienda el uso de tratamiento antibiótico profiláctico ni tratamiento crónico vía oral (16, 18).

\section{a. e Moduladores CFTR:}

Este tratamiento está dirigido a mejorar la producción, procesamiento intracelular y función de la proteína CFTR; estudios han evidenciado que estos medicamentos mejoran el VEF $\square$, disminuyen los síntomas y las exacerbaciones pulmonares $(2,9,19)$. Dependiendo del tipo de fármaco, pueden ser correctores o potenciadores de la proteína, de ahí la importancia del estudio genético para valorar si son candidatos al tratamiento con moduladores del CFTR (19).

Los medicamentos moduladores del CFTR descubiertos hasta ahora son el ivacaftor, lumacaftor, tezacaftor y elexacaftor, los mismos se usan en monoterapia o combinados y, su uso depende del tipo de mutación que posee el paciente.

\section{b. Tratamiento Nutricional}

Se ha demostrado que existe una relación proporcional entre el índice de masa corporal (IMC) y el VEF ${ }_{1}(6,13)$, por lo tanto, es importante mantener un adecuado estado nutricional, el cual se debe vigilar en todas las visitas médicas de control. La meta del tratamiento nutricional es mantener un IMC sobre el percentil 50 en pacientes pediátricos mayores de 2 años, en niños menores de dos años se utiliza gráficas de peso/talla, en mujeres adultas un IMC sobre $22 \mathrm{~kg} / \mathrm{m}^{2}$ y hombres adultos sobre $23 \mathrm{~kg} / \mathrm{m}^{2}(6$, $12,13)$.

Para alcanzar las metas de peso y talla en los pacientes con $F Q$, se recomienda un régimen con $150 \%$ de calorías que ocuparía una persona sana de la misma edad, la distribución de estas calorías debe ser $20 \%$ proteínas, $40-45 \%$ carbohidratos y $35-40 \%$ grasas $(6,12,13)$. La recomendación de lactancia materna exclusiva es la misma que para pacientes sin enfermedad, hasta los 6 meses, la lactancia materna se ha asociado con mejor función pulmonar y menos exacerbaciones (13).

La terapia de reemplazo con enzimas pancreáticas se inicia en pacientes con alta sospecha de insuficiencia pancreática o con diagnóstico confirmado (20). La dosificación de enzimas pancreáticas varía según la cantidad de grasas consumidas por comida o diariamente, el aspecto de las heces y el estado nutricional del paciente (6). No se debe de exceder la dosificación enzimática a $2500 \mathrm{U} / \mathrm{kg}$ por comida o $1000 \mathrm{U} / \mathrm{kg}$ por día, dado el riesgo de desarrollar colopatía fibrosante $(6,13,20)$.

Los suplementos de vitaminas liposolubles se deben iniciar tan pronto como se diagnostique la $F Q$, aún en pacientes asintomáticos o con 
suficiencia pancreática. La vitamina A rara vez es insuficiente en pacientes con FQ; sin embargo, se debe prestar especial atención a la toxicidad que puede provocar la suplementación de dicha vitamina (13). Con respecto a la vitamina $D$, su deficiencia es frecuente en estos pacientes y contribuye al empeoramiento de la salud ósea, asociado a la malabsorción de minerales como el calcio y magnesio $(12,13,20)$. La vitamina E neutraliza los radicales libres de oxígeno, se cree que la deficiencia puede promover el estado inflamatorio y el empeoramiento de la enfermedad pulmonar en pacientes con fibrosis quística $(12,13)$. La deficiencia de vitamina $\mathrm{K}$ es frecuente en pacientes con fibrosis quística, en más del $50 \%$ de los casos (12), esta provoca alteraciones de la coagulación y empeora la enfermedad ósea (13); esta deficiencia no es solamente provocada por la malabsorción de grasas, sino también por el sobrecrecimiento bacteriano en el intestino, el uso constante de antibióticos, la enfermedad hepática y resecciones de intestino.

\section{CONCLUSIONES}

La fibrosis quística (FQ) es la enfermedad genética autosómica recesiva que afecta con mayor frecuencia a la población caucásica, previamente era reconocida como una patología pediátrica de pobre pronóstico, sin embargo, gracias a los avances realizados en el diagnóstico y tratamiento, la supervivencia se ha mostrado en aumento con el paso de los años.

El CFTR es la proteína mutada en esta enfermedad, ella está presente en muchos órganos, esto convierte a la $F Q$ en una enfermedad multisistémica, donde las manifestaciones clínicas mayores se observan en tracto respiratorio y gastrointestinal. El cuadro clínico respiratorio puede ir desde lactantes asintomáticos hasta pacientes con patrones pulmonares obstructivos o restrictivos en fases avanzadas, además, se encuentra la colonización bacteriana crónica característica asociada a inflamación, estas últimas conducen a un proceso de destrucción tisular progresiva, lo cual convierte la enfermedad pulmonar en la principal causa de morbimortalidad en pacientes con FQ. Con respecto al tracto gastrointestinal, la insuficiencia pancreática genera desnutrición, deficiencias vitamínicas y retraso del crecimiento y desarrollo; por otro lado, se describe íleo meconial en recién nacidos, obstrucción intestinal, enfermedad hepatobiliar y otras menos frecuentes. Existen manifestaciones clínicas en otros sistemas, además, por ejemplo, infertilidad, disminución de densidad ósea y algunas secundarias a los cuadros clínicos antes mencionados, tal como incontinencia urinaria por tos crónica.

Para la mejoría de la supervivencia, han sido fundamentales los avances realizados en el diagnóstico, lo cual ha ocurrido principalmente tras la sospecha de fibrosis quística por medio del tamizaje neonatal, donde se realiza la medición de tripsinógeno inmunoreactivo. Esta prueba actualmente ha conducido al diagnóstico de la mayoría de los pacientes con FQ; sin embargo, cabe aclarar que una prueba positiva de tripsinógeno inmunoreactivo, debe confirmarse con análisis de ADN para detectar las mutaciones correspondientes $y$ debe realizarse el test de sudor.

Finalmente, el tratamiento y sus nuevos descubrimientos de igual manera han sido esenciales para prolongar la vida de los pacientes. Este se basa en establecer un 
abordaje multidisciplinario, empezando por los dos factores que generan disminución en la morbimortalidad: tratamiento respiratorio y tratamiento nutricional. El tratamiento respiratorio incluye medios físicos para ayudar a la eliminación de secreciones, medicamentos inhalados para disminuir la viscosidad de las secreciones respiratorias, además se requieren broncodilatadores, antiinflamatorios, terapia antibiótica para exacerbaciones e infección crónica, también se describe el uso de moduladores CTFR. La terapia gastrointestinal radica en el reemplazo de enzimas pancreáticas y el uso de suplementos de vitaminas liposolubles.

\section{REFERENCIAS}

1. Egan, M. E., Schechter, M. S., Voynow, J. A. Cystic Fibrosis. En: Kliegman, R. M., St. Geme, J. Nelson Textbook of Pediatrics. Edición 21. Elsevier Health Sciences; 2019 [consultado 2 abril 2020]; pp. 9016-9065

2. Gartner, S., Mondéjar-López, P., Asensio de la Cruz, O., Grupo de Trabajo de Fibrosis Quística de la Sociedad Española de Neumología Pediátrica. Protocolo de seguimiento de pacientes con fibrosis quística diagnosticados por cribado neonatal. Anales de Pediatría. 2019 [consultado 15 abril 2020]; 90(4): 251.e1-251.e10. Disponible en: https://www.analesdepediatria.org/ es-pdf-S1695403318305393

3. C. Férec V. Scotet. Genetics of cystic fibrosis: Basics. Archives de Pédiatrie.2020 [consultado 2 abril 2020]; 27(1): e54-e57. Disponible en: https://www.sciencedirect.com/science/article/abs/pii/ S0929693X20300439

4. Ruíz de Valbuena, M. Fibrosis quística y sus manifestaciones respiratorias. Pediatría Integral. 2016 [consultado 8 abril 2020]; XX (2): 119-127. Disponible en: https://www.pediatriaintegral.es/ wp-content/uploads/2016/xx02/05/n2-119-127_MartaRuiz.pdf

5. Wiencek, J. R., Lo, S. F. Advances in the diagnostic and management of Cystic Fibrosis en the Genomic Era. Clinical Chemistry. 2018 [consultado 4 abril 2020]; (64)6: 898-908. Disponible en: https://pubmed.ncbi.nlm.nih.gov/29436379/

6. Fielbaum, O. Manejo actual de la Fibrosis Quística. Rev. Med. Clin. Condes. 2017 [consultado 6 abril 2020]; 28(1): 60-71. Disponible en:https://www.elsevier.es/es-revista-revista-medica-clinica-las-condes-202-pdf-S0716864017300159

7. Katkin, J. O. Cystic fibrosis: Genetics and pathogenesis. UpToDate [Internet]. 2020 [consultado 15 abril 2020]. Disponible en: https://www.uptodate.com/contents/cystic-fibrosis-genetics-and-pathogenesis 
8. Sorscher, E. Cystic Fibrosis. En: Jameson, J. L., Fauci, A., Kasper, D., Hauser, S., Longo, D., Loscalzo, J. Harrison's Principles of Internal Medicine. Edición 20. New York: McGraw Hill; 2020 [consultado 2 abril 2020]; pp. 1986-1989

9. Mendoza Chávez, M.M. Manifestaciones respiratorias en la Fibrosis Quística. Adolescere, 2018; 6(3): 51.e1-52. e8. Disponible en: https://www.adolescenciasema.org/ficheros/REVISTA\%20 ADOLESCERE/vol6num3-2018/52.e1-52.e8-manifestaciones\%20respiratorias $\% 20$ fibrosis $\% 20$ quistica.pdf

10. Katkin, J. P. Cystic fibrosis: Clinical manifestations and diagnosis. UpToDate [Internet]. 2020 [consultado 15 abril 2020]. Disponible en: https://www.uptodate.com/contents/cystic-fibrosis-clinical-manifestations-and-diagnosis

11. Simon, R. H. Cystic fibrosis: Overview of the treatment of lung disease. UpToDate [Internet]. 2020 [consultado 15 abril 2020]. Disponible en: https://www.uptodate.com/contents/cystic-fibrosis-overview-of-the-treatment-of-lung-disease

12. Contreras Bolívar, V., Olveira Fuster, C., Blasco Alonso, J., Olveira Fuster, G. Actualización en nutrición en la fibrosis quística. Nutrición Clínica en Medicina. 2019 [consultado 25 abril 2020]; 13(1): 19-44. Disponible en:http://www.aulamedica.es/nutricionclinicamedicina/pdf/5071.pdf

13. Baker, R. D., Baker, S. S. Cystic fibrosis: Nutricional issues. UpToDate [Internet]. 2020 [consultado 22 abril 2020]. Disponible en: https://www.uptodate.com/contents/cystic-fibrosis-nutritional-issues

14. Sabharwal, S., Schwarzenberg, S. J. Cystic fibrosis: Overview of gastrointestinal disease. UpToDate [Internet]. 2020 [consultado 20 abril 2020]. Disponible en: https://www.uptodate.com/ contents/cystic-fibrosis-overview-of-gastrointestinal-disease

15. Cystic fibrosis sweat test. DynaMed [Internet]. 2019 [consultado 30 abril 2020]. Disponible en: https://www.dynamed.com/lab-monograph/cystic-fibrosis-sweat-test

16. Pizarro, M. E., Espinoza-Palma, T. Tratamiento de la Fibrosis Quística: pasado y presente. Neumología Pediátrica. 2016 [consultado 22 abril 2020]; 11(1): 38-43. Disponible en: https://www. neumologia-pediatrica.cl/wp-content/uploads/2017/07/tratamiento-fq.pdf

17. Simon, R. H. Cystic fibrosis: Treatment of acute pulmonary exacerbations. UpToDate [Internet]. 2020 [consultado 02 mayo 2020]. Disponible en: https://www.uptodate.com/contents/cystic-fibrosis-treatment-of-acute-pulmonary-exacerbations 
18. Simon, R. H. Cystic fibrosis: Antibiotic therapy for chronic pulmonary infection. UpToDate [Internet]. 2020 [consultado 02 mayo 2020]. Disponible en: https://www.uptodate.com/contents/ cystic-fibrosis-antibiotic-therapy-for-chronic-pulmonary-infection [Accesado 08 enero 2020]

19. Simon, R. H. Cystic fibrosis: Treatment with CFTR modulators. UpToDate [Internet]. 2020 [consultado 02 mayo 2020]. Disponible en: https://www.uptodate.com/contents/cystic-fibrosis-treatment-with-cftr-modulators

20. Katkin, J. P., Baker, R. D., Baker, S. S. Cystic fibrosis: Assessment and management of pancreatic insufficiency. UpToDate [Internet]. 2020 [consultado 02 mayo 2020]. Disponible en: https://www.uptodate.com/contents/cystic-fibrosis-assessment-and-management-of-pancreatic-insufficiency [Accesado 05 junio 2019] 